\title{
On the asymptotic behavior of fourth-order functional differential equations
}

Osama Moaaz ${ }^{1}$, Elmetwally M Elabbasy ${ }^{1}$ and Omar Bazighifan²*

"Correspondence: o.bazighifan@gmail.com

${ }^{2}$ Department of Mathematics, Hadhramout University,

Hadhramout, Yemen

Full list of author information is

available at the end of the article

\begin{abstract}
The aim of this work is to study asymptotic properties of a class of fourth-order delay differential equations. Our results in this paper not only generalize some previous results, but also improve the earlier ones. Examples are considered to elucidate the main results.
\end{abstract}

Keywords: asymptotic behavior; fourth-order; delay differential equations

\section{Introduction}

This paper is concerned with the oscillatory behavior of solutions of nonlinear fourthorder differential equations of the type

$$
\left(r(\tau)\left(x^{\prime \prime \prime}(\tau)\right)^{\alpha}\right)^{\prime}+\int_{a}^{b} q(\tau, \xi) f(x(g(\tau, \xi))) d \sigma(\xi)=0,
$$

where the following conditions are satisfied:

$\left(\mathrm{A}_{1}\right) \quad r \in C\left(\left[\tau_{0}, \infty\right),(0, \infty)\right), r^{\prime}(\tau)>0$ and $\alpha$ is a quotient of odd positive integers;

$\left(\mathrm{A}_{2}\right) q, g \in C\left(\left[\tau_{0}, \infty\right) \times[a, b], \mathbb{R}\right), q(\tau, \xi) \geq 0, q(\tau, \xi)$ is not zero on any half line $\left[\tau_{\lambda}, \infty\right) \times$ $[a, b], \tau_{\lambda} \geq \tau_{0}, g(\tau, \xi) \leq \tau$ for $\tau \geq \tau_{0}$ and $\xi \in[a, b], g(\tau, \xi)$ is continuous, nondecreasing with respect to $\xi$ and $\lim _{\tau \rightarrow \infty} g(\tau, \xi)=\infty$;

$\left(\mathrm{A}_{3}\right) \sigma \in C([a, b], \mathbb{R}), \sigma$ is nondecreasing and the integral of equation (1.1) is in the Riemann-Stieltjes sense;

and the function $f \in C(\mathbb{R}, \mathbb{R})$ satisfies one of the following conditions:

$\left(\mathrm{S}_{1}\right) f(x) / x^{\alpha} \geq k_{1}>0$ for $x \neq 0$;

$\left(\mathrm{S}_{2}\right) f^{\prime}(x) /|f(x)|^{\frac{1-\alpha}{\alpha}} \geq k_{2}>0$ for $x \neq 0$ and $f(u v) \geq u^{\alpha} f(v)$ for $u v>0$.

By a solution of equation (1.1), we mean a function $x(\tau) \in C\left[\tau_{x}, \infty\right), \tau_{x} \geq \tau_{0}$ such that $r(\tau)\left(x^{\prime \prime \prime}(\tau)\right)^{\alpha}$ is continuously differentiable for all $\tau \geq \tau_{x}$ and satisfies equation (1.1) for all $\tau \in\left[\tau_{x}, \infty\right)$. Here, we consider only proper solutions $x(\tau)$ to equation (1.1) with property $\sup \{|x(\tau)|: \tau \geq \tau\}>0$ for any $\tau \geq \tau_{x}$. A solution of equation (1.1) is called oscillatory if it has arbitrary large zeros, otherwise it is called nonoscillatory.

In recent years there has been much research activity concerning the oscillation behavior of solutions of nonlinear differential equations (see [1-21]). In the last few years, many 
papers have appeared on the oscillatory theory of fourth-order differential equations (see $[2,16,22-25])$.

The aim of this paper is to study the oscillatory behavior of the solutions of nonlinear fourth-order differential equations (1.1) under the assumption

$$
\int_{\tau_{0}}^{\infty} \frac{1}{r^{1 / \alpha}(s)} d s<\infty
$$

and we consider the function $f$ with and without monotonicity. The results obtained essentially generalize the results from Zhang [24] and also improve some results from Baculykova [2]. Examples are provided to illustrate new results.

In order to discuss our main results, we need the following lemmas.

Lemma 1.1 ([15]) If the function y satisfies $y^{(i)}(\tau)>0, i=0,1, \ldots, n$, and $y^{(n+1)}(\tau)<0$, then

$$
\frac{y(\tau)}{\tau^{n} / n !} \geq \frac{y^{\prime}(\tau)}{\tau^{n-1} /(n-1) !} .
$$

Lemma $1.2([1])$ Let $y \in C^{n}\left(\left[\tau_{0}, \infty\right),(0, \infty)\right)$. Assume that $y^{(n)}(\tau)$ is of fixed sign and not identically zero on $\left[\tau_{0}, \infty\right)$ and that there exists $\tau_{1} \geq \tau_{0}$ such that $y^{(n-1)}(\tau) y^{(n)}(\tau) \leq 0$ for all $\tau \geq \tau_{1}$. If $\lim _{\tau \rightarrow \infty} y(\tau) \neq 0$, then for every $\mu \in(0,1)$ there exists $\tau_{\mu} \geq \tau_{1}$ such that

$$
y(\tau) \geq \frac{\mu}{(n-1) !} \tau^{n-1}\left|y^{(n-1)}(\tau)\right| \quad \text { for } \tau \geq \tau_{\mu} .
$$

\section{Main results}

In this section, we establish new oscillation criteria for solutions of equation (1.1). For the sake of convenience, we insert the following notation:

$$
\begin{aligned}
& R_{1}(\tau)=\int_{\tau}^{\infty} \frac{1}{r^{1 / \alpha}(s)} d s, \quad R_{i}(\tau)=\int_{\tau}^{\infty} R_{i-1}(u) d u, \quad i=2,3 \\
& Q(\tau)=\int_{a}^{b} q(\tau, \xi) d \sigma(\xi),
\end{aligned}
$$

and $F_{+}(\tau)=\max \{0, F(\tau)\}$.

Lemma 2.1 If $x(\tau)$ is an eventually positive three times continuously differentiable function such that $r(\tau) x^{\prime \prime \prime}(\tau)$ is continuously differentiable and $\left(r(\tau) x^{\prime \prime \prime}(\tau)\right)^{\prime} \leq 0$ for large $t$, then one of the following cases holds for large $t$ :

$$
\begin{aligned}
& \left(\mathrm{C}_{1}\right) \quad x^{\prime}(\tau)>0, \quad x^{\prime \prime}(\tau)>0, \quad x^{\prime \prime \prime}(\tau)>0, \\
& \left(\mathrm{C}_{2}\right) \quad x^{\prime}(\tau)>0, \quad x^{\prime \prime}(\tau)<0, \quad x^{\prime \prime \prime}(\tau)>0, \\
& \left(\mathrm{C}_{3}\right) \quad x^{\prime}(\tau)<0, \quad x^{\prime \prime}(\tau)>0, \quad x^{\prime \prime \prime}(\tau)<0, \\
& \left(\mathrm{C}_{4}\right) \quad x^{\prime}(\tau)>0, \quad x^{\prime \prime}(\tau)>0, \quad x^{\prime \prime \prime}(\tau)<0 .
\end{aligned}
$$

The proof is immediate and hence is omitted. 
Theorem 2.1 Assume that (1.2) and $\left(\mathrm{S}_{1}\right)$ hold. If there exist continuously differentiable functions $\rho, \vartheta \in C\left(\left[\tau_{0}, \infty\right),(0, \infty)\right)$ such that

$$
\begin{aligned}
& \int_{\tau_{0}}^{\infty}\left(k_{1} \rho(s) Q(s)\left(\frac{g(s, a)}{s}\right)^{3 \alpha}-\frac{2^{\alpha}}{(\alpha+1)^{\alpha+1}} \frac{r(s)\left(\rho_{+}^{\prime}(s)\right)^{\alpha+1}}{\left(\mu_{1} s^{2} \rho(s)\right)^{\alpha}}\right) d s=\infty, \\
& \int_{\tau_{0}}^{\infty}\left(\vartheta(u) \int_{u}^{\infty}\left[\frac{k_{1}}{r(v)} \int_{v}^{\infty} \int_{a}^{b} q(s, \xi) \frac{g^{\alpha}(s, \xi)}{s^{\alpha}} d \sigma(\xi) d s\right]^{1 / \alpha} d v-\frac{\vartheta_{+}^{\prime 2}(u)}{4 \vartheta(u)}\right) d u=\infty, \\
& \int_{\tau_{0}}^{\infty}\left(k_{1} R_{3}^{\alpha}(s) Q(s)-\left(\frac{\alpha}{\alpha+1}\right)^{\alpha+1} \frac{R_{2}(s)}{R_{3}(s)}\right) d s=\infty
\end{aligned}
$$

and

$$
\int_{\tau_{0}}^{\infty}\left(\frac{k_{1} \mu_{2}^{\alpha}}{2^{\alpha}} R_{1}^{\alpha}(s) g^{2 \alpha}(s, a) Q(s)-\left(\frac{\alpha}{\alpha+1}\right)^{\alpha+1} \frac{1}{r^{1 / \alpha}(s) R_{1}(s)}\right) d s=\infty
$$

for some $\mu_{1}, \mu_{2} \in(0,1)$, then every solution of $(1.1)$ is oscillatory.

Proof Let $x$ be a nonoscillatory solution of equation (1.1) on the interval $\left[\tau_{0}, \infty\right)$. Without loss of generality, we may assume that $x(\tau)>0$. From Lemma 2.1, there exists $\tau_{1} \geq \tau_{0}$ such that $x(\tau)$ has one of the four cases $\left(C_{1}\right)-\left(C_{4}\right)$ for $\tau \geq \tau_{1}$. For Case $\left(C_{1}\right)$, we define

$$
\omega(\tau)=\rho(\tau) \frac{r(\tau)\left(x^{\prime \prime \prime}(\tau)\right)^{\alpha}}{x^{\alpha}(\tau)}
$$

Then $\omega(\tau)>0$. By differentiating, we obtain

$$
\omega^{\prime}(\tau)=\frac{\rho^{\prime}(\tau)}{\rho(\tau)} \omega(\tau)+\rho(\tau) \frac{\left(r(\tau)\left(x^{\prime \prime \prime}(\tau)\right)^{\alpha}\right)^{\prime}}{x^{\alpha}(\tau)}-\alpha \rho(\tau) \frac{r(\tau)\left(x^{\prime \prime \prime}(\tau)\right)^{\alpha}}{x^{\alpha+1}(\tau)} x^{\prime}(\tau) .
$$

It follows from Lemma 1.2 that

$$
x^{\prime}(\tau) \geq \frac{\mu}{2} \tau^{2} x^{\prime \prime \prime}(\tau)
$$

for all $\mu \in(0,1)$ and every sufficiently large $\tau$. From $(1.1),\left(\mathrm{A}_{2}\right)$ and $\left(\mathrm{S}_{1}\right)$, we see that

$$
\begin{aligned}
\left(r(\tau)\left(x^{\prime \prime \prime}(\tau)\right)^{\alpha}\right)^{\prime} & =-\int_{a}^{b} q(\tau, \xi) f(x(g(\tau, \xi))) d \sigma(\xi) \\
& \leq-k_{1} Q(\tau) x^{\alpha}(g(\tau, a)) .
\end{aligned}
$$

Thus, by (2.5), (2.6) and (2.7), we get

$$
\omega^{\prime}(\tau) \leq \frac{\rho^{\prime}(\tau)}{\rho(\tau)} \omega(\tau)-k_{1} \rho(\tau) Q(\tau) \frac{x^{\alpha}(g(\tau, a))}{x^{\alpha}(\tau)}-\frac{\alpha \mu}{2} \tau^{2} \rho(\tau) r(\tau)\left(\frac{x^{\prime \prime \prime}(\tau)}{x(\tau)}\right)^{\alpha+1} .
$$

From Lemma 1.1, we have that

$$
x(\tau) \geq \frac{\tau}{3} x^{\prime}(\tau) .
$$


Integrating this inequality from $g(\tau, a)$ to $\tau$, we get

$$
\frac{x(g(\tau, a))}{x(\tau)} \geq \frac{g^{3}(\tau, a)}{\tau^{3}}
$$

which with (2.8) gives

$$
\omega^{\prime}(\tau) \leq \frac{\rho^{\prime}(\tau)}{\rho(\tau)} \omega(\tau)-k_{1} \rho(\tau) Q(\tau)\left(\frac{g(\tau, a)}{\tau}\right)^{3 \alpha}-\frac{\alpha \mu}{2} \frac{\tau^{2}}{(\rho(\tau) r(\tau))^{1 / \alpha}} \omega^{\frac{\alpha+1}{\alpha}}(\tau) .
$$

By using the inequality

$$
B z-A z^{\frac{1+\alpha}{\alpha}} \leq \frac{\alpha^{\alpha}}{(\alpha+1)^{\alpha+1}} B^{\alpha+1} A^{-\alpha} \quad \text { for } A, B>0 \text { and } z \geq 0,
$$

with $A=\frac{\alpha \mu}{2} \frac{\tau^{2}}{(\rho(\tau) r(\tau))^{1 / \alpha}}, B=\frac{\rho^{\prime}(\tau)}{\rho(\tau)}$ and $z=\omega$, we get

$$
\omega^{\prime}(\tau) \leq-k_{1} \rho(\tau) Q(\tau)\left(\frac{g(\tau, a)}{\tau}\right)^{3 \alpha}+\frac{2^{\alpha}}{(\alpha+1)^{\alpha+1}} \frac{r(\tau)\left(\rho_{+}^{\prime}(\tau)\right)^{\alpha+1}}{\left(\mu \tau^{2} \rho(\tau)\right)^{\alpha}} .
$$

This implies that

$$
\int_{\tau_{1}}^{\tau}\left(k_{1} \rho(s) Q(s)\left(\frac{g(s, a)}{s}\right)^{3 \alpha}-\frac{2^{\alpha}}{(\alpha+1)^{\alpha+1}} \frac{r(s)\left(\rho_{+}^{\prime}(\tau)\right)^{\alpha+1}}{\left(\mu s^{2} \rho(s)\right)^{\alpha}}\right) d s \leq \omega\left(\tau_{1}\right)
$$

for every $\mu \in(0,1)$ and all sufficiently large $\tau$, which contradicts (2.1).

Consider Case $\left(\mathrm{C}_{2}\right)$ holds. From Lemma 1.1, we get that $x(\tau) \geq \tau x^{\prime}(\tau)$, by integrating this inequality from $g(\tau, \xi)$ to $\tau$, we get

$$
x(g(\tau, \xi)) \geq \frac{g(\tau, \xi)}{\tau} x(\tau)
$$

Hence, from $\left(S_{1}\right)$, we have

$$
f(x(g(\tau, \xi))) \geq k_{1} \frac{g^{\alpha}(s, \xi)}{s^{\alpha}} x^{\alpha}(\tau) .
$$

Integrating (1.1) from $\tau$ to $u$ and using $x^{\prime}(\tau)>0$, we obtain

$$
\begin{aligned}
r(u)\left(x^{\prime \prime \prime}(u)\right)^{\alpha}-r(\tau)\left(x^{\prime \prime \prime}(\tau)\right)^{\alpha} & =-\int_{\tau}^{u} \int_{a}^{b} q(s, \xi) f(x(g(s, \xi))) d \sigma(\xi) d s \\
& \leq-k_{1} x^{\alpha}(\tau) \int_{\tau}^{u} \int_{a}^{b} q(s, \xi) \frac{g^{\alpha}(s, \xi)}{s^{\alpha}} d \sigma(\xi) d s .
\end{aligned}
$$

Letting $u \rightarrow \infty$, we see that

$$
r(\tau)\left(x^{\prime \prime \prime}(\tau)\right)^{\alpha} \geq k_{1} x^{\alpha}(\tau) \int_{\tau}^{\infty} \int_{a}^{b} q(s, \xi) \frac{g^{\alpha}(s, \xi)}{s^{\alpha}} d \sigma(\xi) d s,
$$

and so,

$$
x^{\prime \prime \prime}(\tau) \geq x(\tau)\left[\frac{k_{1}}{r(\tau)} \int_{\tau}^{\infty} \int_{a}^{b} q(s, \xi) \frac{g^{\alpha}(s, \xi)}{s^{\alpha}} d \sigma(\xi) d s\right]^{1 / \alpha} .
$$


Integrating again from $\tau$ to $\infty$, we get

$$
x^{\prime \prime}(\tau) \leq-x(\tau) \int_{\tau}^{\infty}\left[\frac{k_{1}}{r(v)} \int_{v}^{\infty} \int_{a}^{b} q(s, \xi) \frac{g^{\alpha}(s, \xi)}{s^{\alpha}} d \sigma(\xi) d s\right]^{1 / \alpha} d v
$$

Now, we define

$$
w(\tau)=\vartheta(\tau) \frac{x^{\prime}(\tau)}{x(\tau)}
$$

Then $w(\tau)>0$ for $\tau \geq \tau_{1}$. By differentiating and using (2.13), we find

$$
\begin{aligned}
w^{\prime}(\tau)= & \frac{\vartheta^{\prime}(\tau)}{\vartheta(\tau)} w(\tau)+\vartheta(\tau) \frac{x^{\prime \prime}(\tau)}{x(\tau)}-\vartheta(\tau) \frac{\left(x^{\prime}(\tau)\right)^{2}}{x^{2}(\tau)} \\
\leq & -\vartheta(\tau) \int_{\tau}^{\infty}\left[\frac{k}{r(v)} \int_{v}^{\infty} \int_{a}^{b} q(s, \xi) \frac{g^{\alpha}(s, \xi)}{s^{\alpha}} d \sigma(\xi) d s\right]^{1 / \alpha} d v \\
& +\frac{\vartheta^{\prime}(\tau)}{\vartheta(\tau)} w(\tau)-\frac{1}{\vartheta(\tau)} w^{2}(\tau) .
\end{aligned}
$$

Thus, we obtain

$$
w^{\prime}(\tau) \leq-\vartheta(\tau) \int_{\tau}^{\infty}\left[\frac{k}{r(v)} \int_{v}^{\infty} \int_{a}^{b} q(s, \xi) \frac{g^{\alpha}(s, \xi)}{s^{\alpha}} d \sigma(\xi) d s\right]^{1 / \alpha} d v+\frac{\left(\vartheta_{+}^{\prime}(\tau)\right)^{2}}{4 \vartheta(\tau)}
$$

Then we get

$$
\begin{aligned}
& \int_{\tau_{1}}^{\tau}\left(\vartheta(u) \int_{u}^{\infty}\left[\frac{k}{r(v)} \int_{v}^{\infty} \int_{a}^{b} q(s, \xi) \frac{g^{\alpha}(s, \xi)}{s^{\alpha}} d \sigma(\xi) d s\right]^{1 / \alpha} d v-\frac{\vartheta_{+}^{\prime 2}(u)}{4 \vartheta(u)}\right) d u \\
& \quad \leq w\left(\tau_{1}\right) .
\end{aligned}
$$

This contradicts (2.2).

Assume that Case $\left(\mathrm{C}_{3}\right)$ holds. Since $r(\tau)\left(x^{\prime \prime \prime}(\tau)\right)^{\alpha}$ is nonincreasing, we have that $r(s)\left(x^{\prime \prime \prime}(s)\right)^{\alpha} \leq r(\tau)\left(x^{\prime \prime \prime}(\tau)\right)^{\alpha}$ for all $s \geq \tau \geq \tau_{1}$. This yields

$$
x^{\prime \prime \prime}(s) \leq\left[r(\tau)\left(x^{\prime \prime \prime}(\tau)\right)^{\alpha}\right]^{1 / \alpha} \frac{1}{r^{1 / \alpha}(s)} .
$$

Integrating this inequality from $\tau$ to $u$, we get

$$
x^{\prime \prime}(u)-x^{\prime \prime}(\tau) \leq\left[r(\tau)\left(x^{\prime \prime \prime}(\tau)\right)^{\alpha}\right]^{1 / \alpha} \int_{\tau}^{u} \frac{1}{r^{1 / \alpha}(s)} d s .
$$

Letting $u \rightarrow \infty$, we see that

$$
-x^{\prime \prime}(\tau) \leq\left[r(\tau)\left(x^{\prime \prime \prime}(\tau)\right)^{\alpha}\right]^{1 / \alpha} R_{1}(\tau)
$$

By integrating the last inequality from $\tau$ to $\infty$, we obtain

$$
x^{\prime}(\tau) \leq\left[r(\tau)\left(x^{\prime \prime \prime}(\tau)\right)^{\alpha}\right]^{1 / \alpha} R_{2}(\tau) .
$$


Integrating again from $\tau$ to $\infty$, we find

$$
x(\tau) \geq-\left[r(\tau)\left(x^{\prime \prime \prime}(\tau)\right)^{\alpha}\right]^{1 / \alpha} R_{3}(\tau)
$$

Next, we define

$$
\psi(\tau)=\frac{r(\tau)\left(x^{\prime \prime \prime}(\tau)\right)^{\alpha}}{x^{\alpha}(\tau)}
$$

Thus, we see that $\psi(\tau)<0$ and satisfies

$$
\psi^{\prime}(\tau)=\frac{\left[r(\tau)\left(x^{\prime \prime \prime}(\tau)\right)^{\alpha}\right]^{\prime}}{x^{\alpha}(\tau)}-\alpha \frac{r(\tau)\left(x^{\prime \prime \prime}(\tau)\right)^{\alpha}}{x^{\alpha+1}(\tau)} x^{\prime}(\tau) .
$$

Hence, from (1.1), (2.17) and $\left(\mathrm{S}_{1}\right)$, we have

$$
\psi^{\prime}(\tau) \leq-k_{1} \int_{a}^{b} q(\tau, \xi) \frac{x^{\alpha}(g(\tau, \xi))}{x^{\alpha}(\tau)} d \sigma(\xi)-\alpha R_{2}(\tau) \psi^{\frac{1+\alpha}{\alpha}}(\tau) .
$$

Since $g(\tau, \xi) \leq \tau$ and $x^{\prime}(\tau)<0$, we have that $x(g(\tau, \xi)) \geq x(\tau)$. Therefore, we get

$$
\psi^{\prime}(\tau) \leq-k_{1} Q(\tau)-\alpha R_{2}(\tau) \psi^{\frac{1+\alpha}{\alpha}}(\tau)
$$

From (2.18), we have

$$
R_{3}^{\alpha}(\tau) \psi(\tau) \geq-1
$$

Multiplying (2.19) by $R_{3}^{\alpha}(\tau)$ and integrating from $\tau_{1}$ to $\tau$, we obtain

$$
\begin{aligned}
& R_{3}^{\alpha}(\tau) \psi(\tau)-R_{3}^{\alpha}\left(\tau_{1}\right) \psi\left(\tau_{1}\right)+\alpha \int_{\tau_{1}}^{\tau} R_{2}(s) R_{3}^{\alpha-1}(s) \psi(s) d s \\
& \quad+k_{1} \int_{\tau_{1}}^{\tau} R_{3}^{\alpha}(s) Q(s) d s+\alpha \int_{\tau_{1}}^{\tau} R_{2}(s) R_{3}^{\alpha}(s) \psi^{\frac{1+\alpha}{\alpha}}(s) d s \leq 0,
\end{aligned}
$$

which with (2.20) gives

$$
\begin{aligned}
1+R_{3}^{\alpha}\left(\tau_{1}\right) \psi\left(\tau_{1}\right) \geq & k_{1} \int_{\tau_{1}}^{\tau} R_{3}^{\alpha}(s) Q(s) d s \\
& +\alpha \int_{\tau_{1}}^{\tau} R_{2}(s) R_{3}^{\alpha-1}(s)\left[\psi(s)+R_{3}(s) \psi^{\frac{1+\alpha}{\alpha}}(s)\right] d s .
\end{aligned}
$$

Using inequality (2.11) with $A=R_{3}, B=1$ and $z=-\psi$, we get

$$
\psi(s)+R_{3}(s) \psi^{\frac{1+\alpha}{\alpha}}(s) \geq-\frac{\alpha^{\alpha}}{(\alpha+1)^{\alpha+1}} R_{3}^{-\alpha}(s) .
$$

It follows that

$$
\int_{\tau_{1}}^{\tau}\left(k_{1} R_{3}^{\alpha}(s) Q(s)-\left(\frac{\alpha}{\alpha+1}\right)^{\alpha+1} \frac{R_{2}(s)}{R_{3}(s)}\right) d s \leq 1+R_{3}^{\alpha}\left(\tau_{1}\right) \psi\left(\tau_{1}\right),
$$

but this contradicts (2.3). 
In Case $\left(C_{4}\right)$. In view of the proof of Case $\left(C_{3}\right)$, we have (2.16) holds. From Lemma 1.2, we have that $x(\tau) \geq \frac{\mu}{2} \tau^{2} x^{\prime \prime}(\tau)$ for all $\mu \in(0,1)$ and every sufficiently large $\tau$. Thus, from $\left(\mathrm{A}_{2}\right)$, there exists $\tau_{2} \geq \tau_{1}$ such that

$$
\frac{x(g(\tau, a))}{x^{\prime \prime}(g(\tau, a))} \geq \frac{\mu}{2} g^{2}(\tau, a)
$$

for $\tau \geq \tau_{2}$. Next, we define

$$
\varphi(\tau)=\frac{r(\tau)\left(x^{\prime \prime \prime}(\tau)\right)^{\alpha}}{\left(x^{\prime \prime}(\tau)\right)^{\alpha}}
$$

We note that $\varphi(\tau)<0$ for $\tau \geq \tau_{1}$. By differentiating and using (1.1), ( $\left.\mathrm{A}_{3}\right)$ and $\left(\mathrm{S}_{1}\right)$, we obtain

$$
\varphi^{\prime}(\tau) \leq-k_{1} Q(\tau) \frac{x^{\alpha}(g(\tau, a))}{\left(x^{\prime \prime}(g(\tau, a))\right)^{\alpha}} \frac{\left(x^{\prime \prime}(g(\tau, a))\right)^{\alpha}}{\left(x^{\prime \prime}(\tau)\right)^{\alpha}}-\alpha \frac{1}{r^{1 / \alpha}(\tau)} \varphi^{\frac{\alpha+1}{\alpha}}(\tau) .
$$

Hence, (2.21) yields

$$
\varphi^{\prime}(\tau) \leq-\frac{k_{1} \mu^{\alpha}}{2^{\alpha}} g^{2 \alpha}(\tau, a) Q(\tau)-\alpha \frac{1}{r^{1 / \alpha}(\tau)} \varphi^{\frac{\alpha+1}{\alpha}}(\tau)
$$

From (2.16), we get

$$
R_{1}^{\alpha}(\tau) \varphi(\tau) \geq-1
$$

Multiplying (2.23) by $R_{1}^{\alpha}(\tau)$ and integrating from $\tau_{2}$ to $\tau$, we obtain

$$
\begin{aligned}
1+R_{1}^{\alpha}\left(\tau_{2}\right) \varphi\left(\tau_{2}\right) \geq & \frac{k_{1} \mu^{\alpha}}{2^{\alpha}} \int_{\tau_{2}}^{\tau} R_{1}^{\alpha}(s) g^{2 \alpha}(s, a) Q(s) d s \\
& +\alpha \int_{\tau_{2}}^{\tau} \frac{R_{1}^{\alpha-1}(s)}{r^{1 / \alpha}(s)}\left(\varphi(s)+R_{1}(s) \varphi^{\frac{1+\alpha}{\alpha}}(s)\right) d s .
\end{aligned}
$$

By following the same steps as in Case $\left(C_{3}\right)$, we get that

$$
\begin{aligned}
& \int_{\tau_{2}}^{\tau}\left(\frac{k_{1} \mu^{\alpha}}{2^{\alpha}} R_{1}^{\alpha}(s) g^{2 \alpha}(s, a) Q(s)-\left(\frac{\alpha}{\alpha+1}\right)^{\alpha+1} \frac{1}{r^{1 / \alpha}(s) R_{1}(s)}\right) d s \\
& \quad \leq 1+R_{1}^{\alpha}\left(\tau_{2}\right) \varphi\left(\tau_{2}\right)
\end{aligned}
$$

which contradicts (2.4). This contradiction completes the proof of Theorem 2.1.

Theorem 2.2 Assume that (1.2) and $\left(\mathrm{S}_{2}\right)$ hold, and let $g(\tau, \xi)$ have a positive partial derivative on $I \times[a, b]$ with respect to $\tau$. If there exist continuously differentiable functions $\rho, \vartheta \in C\left(\left[\tau_{0}, \infty\right),(0, \infty)\right)$ such that

$$
\begin{aligned}
& \int_{\tau_{0}}^{\infty}\left(\rho(s) Q(s)-\frac{(2 \alpha)^{\alpha}}{(\alpha+1)^{\alpha+1}} \frac{r(s)\left(\rho_{+}^{\prime}(s)\right)^{\alpha+1}}{\left(\mu k_{2} s^{2} \rho(s) g^{\prime}(s, a)\right)^{\alpha}}\right) d s=\infty \\
& \int_{\tau_{0}}^{\infty}\left(\vartheta(u) \int_{u}^{\infty}\left(\frac{1}{r(v)} \int_{v}^{\infty} Q(s) d s\right)^{1 / \alpha} d v-\frac{\alpha}{4 k_{2}} \frac{\vartheta_{+}^{\prime 2}(u)}{g^{\prime}(u, a) \vartheta(u)}\right) d u=\infty
\end{aligned}
$$




$$
\int_{\tau_{0}}^{\infty}\left(f\left(R_{3}(s)\right) Q(s)-\frac{\alpha^{\alpha}}{(\alpha+1)^{\alpha+1}} R_{2}(s) \frac{\left(f^{\prime}\left(R_{3}(s)\right)\right)^{\alpha+1}}{k_{2}^{\alpha} f^{\alpha}\left(R_{3}(s)\right)}\right) d s=\infty,
$$

and

$$
\int_{\tau_{0}}^{\infty}\left(R_{1}^{\alpha}(s) f\left(\frac{\mu}{2} g^{2}(\tau, a)\right) Q(s)-\left(\frac{\alpha}{\alpha+1}\right)^{\alpha+1} \frac{1}{r^{1 / \alpha}(s) R_{1}(s)}\right) d s=\infty
$$

for some $\mu_{1}, \mu_{2} \in(0,1)$, then every solution of $(1.1)$ is oscillatory.

Proof Let $x$ be a nonoscillatory solution of equation (1.1). Without loss of generality, we may assume that $x(\tau)>0$. By Lemma 2.1, there exists $\tau_{1} \geq \tau_{0}$ such that $x(\tau)$ has one of the four cases $\left(\mathrm{C}_{1}\right)-\left(\mathrm{C}_{4}\right)$ for $\tau \geq \tau_{1}$. For Case $\left(\mathrm{C}_{1}\right)$, since $g(\tau, \xi)$ is nondecreasing with respect to $\xi, x^{\prime}(\tau)>0$ and $f^{\prime}(x)>0$, we have that $f(x(g(\tau, a))) \leq f(x(g(\tau, \xi)))$. Thus, from (1.1), we get

$$
\frac{\left(r(\tau)\left(x^{\prime \prime \prime}(\tau)\right)^{\alpha}\right)^{\prime}}{f(x(g(\tau, a)))} \leq-Q(\tau)
$$

Now, we define

$$
\omega(\tau)=\rho(\tau) \frac{r(\tau)\left(x^{\prime \prime \prime}(\tau)\right)^{\alpha}}{f(x(g(\tau, a)))}
$$

By differentiating and using $\left(\mathrm{S}_{2}\right)$, we get

$$
\begin{aligned}
\omega^{\prime}(\tau) \leq & \frac{\rho^{\prime}(\tau)}{\rho(\tau)} \omega(\tau)-\rho(\tau) Q(\tau) \\
& -k_{2} \rho(\tau) \frac{r(\tau)\left(x^{\prime \prime \prime}(\tau)\right)^{\alpha}}{f^{1+1 / \alpha}(x(g(\tau, a)))} x^{\prime}(g(\tau, a)) g^{\prime}(\tau, a) .
\end{aligned}
$$

From $\left(\mathrm{A}_{2}\right)$, there exists $\tau_{2} \geq \tau_{1}$ such that $g(\tau, a) \geq \tau_{1}$ for $\tau \geq \tau_{2}$. Hence, from Lemma 1.2 and $x^{(4)}<0$, we obtain

$$
x^{\prime}(g(\tau, a)) \geq \frac{\mu}{2} \tau^{2} x^{\prime \prime \prime}(g(\tau, a)) \geq \frac{\mu}{2} \tau^{2} x^{\prime \prime \prime}(\tau)
$$

for all $\mu \in(0,1)$ and $\tau \geq \tau_{2}$. Therefore, (2.28) yields

$$
\omega^{\prime}(\tau) \leq \frac{\rho^{\prime}(\tau)}{\rho(\tau)} \omega(\tau)-\rho(\tau) Q(\tau)-\frac{\mu k_{2}}{2} \frac{\tau^{2} g^{\prime}(\tau, a)}{(\rho(\tau) r(\tau))^{1 / \alpha}} \omega^{\frac{\alpha+1}{\alpha}}(\tau)
$$

By following the same steps as in Case $\left(C_{1}\right)$ of the proof of Theorem 2.1, we get a contradiction with (2.24).

For Case $\left(\mathrm{C}_{2}\right)$. From $(1.1),\left(\mathrm{S}_{2}\right)$ and $\left(\mathrm{A}_{3}\right)$, we obtain

$$
\left(r(\tau)\left(x^{\prime \prime \prime}(\tau)\right)^{\alpha}\right)^{\prime} \leq-f(x(g(\tau, a))) Q(\tau) .
$$

By integrating this inequality from $\tau$ to $\infty$, we obtain

$$
r(\tau)\left(x^{\prime \prime \prime}(\tau)\right)^{\alpha} \geq \int_{\tau}^{\infty} f(x(g(s, a))) Q(s) d s .
$$


Since $f^{\prime}(x)>0$, we get

$$
x^{\prime \prime \prime}(\tau) \geq f^{1 / \alpha}(x(g(\tau, a)))\left(\frac{1}{r(\tau)} \int_{\tau}^{\infty} Q(s) d s\right)^{1 / \alpha} .
$$

Integrating again from $\tau$ to $\infty$, we have

$$
x^{\prime \prime}(\tau) \leq-f^{1 / \alpha}(x(g(\tau, a))) \int_{\tau}^{\infty}\left(\frac{1}{r(v)} \int_{v}^{\infty} Q(s) d s\right)^{1 / \alpha} d \nu
$$

Next, we define

$$
w(\tau)=\vartheta(\tau) \frac{x^{\prime}(\tau)}{f^{1 / \alpha}(x(g(\tau, a)))} .
$$

Then $w(\tau)>0$ for $\tau \geq \tau_{1}$. By differentiating and using (2.13), we find

$$
\begin{aligned}
w^{\prime}(\tau) \leq & \frac{\vartheta^{\prime}(\tau)}{\vartheta(\tau)} w(\tau)-\vartheta(\tau) \int_{\tau}^{\infty}\left(\frac{1}{r(v)} \int_{v}^{\infty} Q(s) d s\right)^{1 / \alpha} d v \\
& -\frac{1}{\alpha} \vartheta(\tau)\left(\frac{x^{\prime}(\tau)}{f^{1 / \alpha}(x(g(\tau, a)))}\right)^{2} \frac{f^{\prime}(x(g(\tau, a)))}{f^{1-\frac{1}{\alpha}}(x(g(\tau, a)))} x^{\prime}(g(\tau, a)) g^{\prime}(\tau, a) .
\end{aligned}
$$

Since $x^{\prime \prime}(\tau)<0$, we see that $x^{\prime}(g(\tau, a))>x^{\prime}(\tau)$

$$
w^{\prime}(\tau) \leq \frac{\vartheta^{\prime}(\tau)}{\vartheta(\tau)} w(\tau)-\vartheta(\tau) \int_{\tau}^{\infty}\left(\frac{1}{r(v)} \int_{v}^{\infty} Q(s) d s\right)^{1 / \alpha} d v-\frac{k_{2}}{\alpha} \frac{g^{\prime}(\tau, a)}{\vartheta(\tau)} w^{2}(\tau) .
$$

Then we get

$$
w^{\prime}(\tau) \leq-\vartheta(\tau) \int_{\tau}^{\infty}\left(\frac{1}{r(v)} \int_{v}^{\infty} Q(s) d s\right)^{1 / \alpha} d v+\frac{\alpha}{4 k_{2}} \frac{\vartheta^{\prime 2}(\tau)}{g^{\prime}(\tau, a) \vartheta(\tau)}
$$

Integrating again from $\tau_{2}$ to $\tau$, we have

$$
\int_{\tau_{2}}^{\tau}\left(\vartheta(u) \int_{u}^{\infty}\left(\frac{1}{r(v)} \int_{v}^{\infty} Q(s) d s\right)^{1 / \alpha} d v-\frac{\alpha}{4 k_{2}} \frac{\vartheta^{\prime 2}(u)}{g^{\prime}(u, a) \vartheta(u)}\right) d u \leq w\left(\tau_{2}\right)
$$

which contradicts (2.25).

If Case $\left(\mathrm{C}_{3}\right)$ holds. As in the proof of Case $\left(\mathrm{C}_{3}\right)$ of Theorem 2.1, we have that (2.16), (2.17) and (2.18) hold. Then we define

$$
\psi(\tau)=\frac{r(\tau)\left(x^{\prime \prime \prime}(\tau)\right)^{\alpha}}{f(x(\tau))}
$$

Thus, we see that $\psi(\tau)<0$ and satisfies

$$
\psi^{\prime}(\tau)=\frac{\left[r(\tau)\left(x^{\prime \prime \prime}(\tau)\right)^{\alpha}\right]^{\prime}}{f(x(\tau))}-\frac{r(\tau)\left(x^{\prime \prime \prime}(\tau)\right)^{\alpha}}{f^{2}(x(\tau))} f^{\prime}(x(\tau)) x^{\prime}(\tau) .
$$


Hence, from (1.1), (2.17) and $\left(\mathrm{S}_{2}\right)$, we have

$$
\psi^{\prime}(\tau) \leq-\int_{a}^{b} q(\tau, \xi) \frac{f(x(g(\tau, \xi)))}{f(x(\tau))} d \sigma(\xi)-k_{2} R_{2}(\tau) \psi^{\frac{1+\alpha}{\alpha}}(\tau) .
$$

Since $x^{\prime}(\tau)<0$, we get $f(x(g(\tau, \xi))) \geq f(x(\tau))$. Therefore, we obtain

$$
\psi^{\prime}(\tau) \leq-Q(\tau)-k_{2} R_{2}(\tau) \psi^{\frac{1+\alpha}{\alpha}}(\tau)
$$

From $(2.18)$ and $\left(\mathrm{S}_{2}\right)$, we have

$$
f\left(R_{3}(\tau)\right) \psi(\tau) \geq-1
$$

Multiplying (2.19) by $f\left(R_{3}(\tau)\right)$ and integrating from $\tau_{1}$ to $\tau$, we obtain

$$
\begin{aligned}
& f\left(R_{3}(\tau)\right) \psi(\tau)-f\left(R_{3}\left(\tau_{1}\right)\right) \psi\left(\tau_{1}\right)+\int_{\tau_{1}}^{\tau} R_{2}(s) f^{\prime}\left(R_{3}(s)\right) \psi(s) d s \\
& \quad+\int_{\tau_{1}}^{\tau} f\left(R_{3}(s)\right) Q(s) d s+k_{2} \int_{\tau_{1}}^{\tau} R_{2}(s) f\left(R_{3}(s)\right) \psi^{\frac{1+\alpha}{\alpha}}(s) d s \leq 0 .
\end{aligned}
$$

Using inequality (2.11) with $A=k_{2} f\left(R_{3}(s)\right), B=f^{\prime}\left(R_{3}(s)\right)$ and $z=-\psi$, we get

$$
\begin{aligned}
& \int_{\tau_{1}}^{\tau}\left(f\left(R_{3}(s)\right) Q(s)-\frac{\alpha^{\alpha}}{(\alpha+1)^{\alpha+1}} R_{2}(s) \frac{\left(f^{\prime}\left(R_{3}(s)\right)\right)^{\alpha+1}}{k_{2}^{\alpha} f^{\alpha}\left(R_{3}(s)\right)}\right) d s \\
& \quad \leq 1+f\left(R_{3}\left(\tau_{1}\right)\right) \psi\left(\tau_{1}\right),
\end{aligned}
$$

but this contradicts (2.26).

In Case $\left(\mathrm{C}_{4}\right)$. In view of the proof of Case $\left(\mathrm{C}_{4}\right)$ of Theorem 2.1, we have (2.16) and (2.21) hold. By defining $\varphi(\tau)$ as the form (2.22), we note that $\varphi(\tau)<0$ for $\tau \geq \tau_{1}$. Thus, from (1.1) and $\left(\mathrm{A}_{2}\right)$, we get

$$
\varphi^{\prime}(\tau) \leq-Q(\tau) \frac{f(x(g(\tau, a)))}{\left(x^{\prime \prime}(g(\tau, a))\right)^{\alpha}} \frac{\left(x^{\prime \prime}(g(\tau, a))\right)^{\alpha}}{\left(x^{\prime \prime}(\tau)\right)^{\alpha}}-\alpha \frac{1}{r^{1 / \alpha}(\tau)} \varphi^{\frac{\alpha+1}{\alpha}}(\tau) .
$$

From $(2.21)$ and $\left(\mathrm{S}_{2}\right)$, we see that

$$
f(x(g(\tau, a))) \geq f\left(\frac{\mu}{2} g^{2}(\tau, a)\right)\left(x^{\prime \prime}(g(\tau, a))\right)^{\alpha} .
$$

Hence, (2.31) yields

$$
\varphi^{\prime}(\tau) \leq-f\left(\frac{\mu}{2} g^{2}(\tau, a)\right) Q(\tau)-\alpha \frac{1}{r^{1 / \alpha}(\tau)} \varphi^{\frac{\alpha+1}{\alpha}}(\tau) .
$$

By following the same steps as in Case $\left(\mathrm{C}_{3}\right)$, we get that

$$
\int_{\tau_{1}}^{\tau}\left(R_{1}^{\alpha}(s) f\left(\frac{\mu}{2} g^{2}(\tau, a)\right) Q(s)-\left(\frac{\alpha}{\alpha+1}\right)^{\alpha+1} \frac{1}{r^{1 / \alpha}(s) R_{1}(s)}\right) d s \leq 1+R_{1}^{\alpha}\left(\tau_{2}\right) \varphi\left(\tau_{2}\right),
$$

which contradicts (2.27). This contradiction completes the proof of Theorem 2.2. 
Theorem 2.3 Assume that (1.2) and $\left(\mathrm{S}_{1}\right)$ hold. If the differential equations

$$
\begin{aligned}
& \left(\frac{r(\tau)}{\tau^{2 \alpha}}\left(x^{\prime}(\tau)\right)^{\alpha}\right)^{\prime}+k_{1} Q(\tau)\left(\frac{\mu_{1} g^{3}(\tau, a)}{2 \tau^{3}}\right)^{\alpha} x^{\alpha}(\tau)=0, \\
& x^{\prime \prime}(\tau)+x(\tau) \int_{\tau}^{\infty}\left[\frac{k_{1}}{r(v)} \int_{v}^{\infty} \int_{a}^{b} q(s, \xi) \frac{g^{\alpha}(s, \xi)}{s^{\alpha}} d \sigma(\xi) d s\right]^{1 / \alpha} d v=0, \\
& \left(\frac{1}{R_{2}^{\alpha}(\tau)}\left(x^{\prime}(\tau)\right)^{\alpha}\right)^{\prime}+k_{1} Q(\tau) x^{\alpha}(\tau)=0
\end{aligned}
$$

and

$$
\left(r(\tau)\left(x^{\prime}(\tau)\right)^{\alpha}\right)^{\prime}+\frac{k_{1} \mu_{2}^{\alpha}}{2^{\alpha}} g^{2 \alpha}(\tau, a) Q(\tau) x^{\alpha}(\tau)=0
$$

are oscillatory for some $\mu_{1}, \mu_{2} \in(0,1)$, then every solution of $(1.1)$ is oscillatory.

Proof Proceeding as in the proof of Theorem 2.1, for Case $\left(C_{1}\right)$, we have that (2.10) holds. Then, if $\rho(\tau)=1$, we get

$$
\omega^{\prime}(\tau)+k_{1} Q(\tau)\left(\frac{g(\tau, a)}{\tau}\right)^{3 \alpha}+\frac{\alpha \mu}{2} \frac{\tau^{2}}{r^{1 / \alpha}(\tau)} \omega^{\frac{\alpha+1}{\alpha}}(\tau) \leq 0
$$

for all $\mu \in(0,1)$. Hence, from [1], we see that (2.32) has a nonoscillatory solution for every $\mu \in(0,1)$, which is a contradiction.

The rest of the proof is the same, and hence is omitted.

From Corollary 1 in Dzurina [3], if

$$
\int_{\tau_{0}}^{\infty} \frac{1}{\widehat{r}^{1 / \alpha}(s)} d s=\infty
$$

and

$$
\liminf _{\tau \rightarrow \infty} \widehat{r}^{1 / \alpha}(\tau)\left[\int_{\tau_{0}}^{\tau} \widehat{r}^{-1 / \alpha}(s) d s\right]^{\alpha+1} \widehat{q}(\tau)>\frac{\alpha}{4},
$$

then equation

$$
\left(\widehat{r}(\tau)\left(u^{\prime}(\tau)\right)^{\alpha}\right)^{\prime}+\widehat{q}(\tau) u^{\alpha}(\tau)=0
$$

is oscillatory. In the following theorem, by using the results of Dzurina [3], we will establish new oscillation criteria for solutions of equation (1.1) under the conditions

$$
\int_{\tau_{0}}^{\infty} \frac{s^{2}}{r^{1 / \alpha}(s)} d s=\infty \quad \text { and } \quad \int_{\tau_{0}}^{\infty} R_{2}(s) d s=\infty
$$

Theorem 2.4 Assume that (1.2), (2.38) and ( $\left.\mathrm{S}_{1}\right)$ hold, and let (2.4) hold for some $\mu_{2} \in(0,1)$. If

$$
\liminf _{\tau \rightarrow \infty} \frac{r^{1 / \alpha}(\tau)}{\tau^{2}}\left(\frac{\mu_{1} g^{3}(\tau, a)}{2 \tau^{3}}\right)^{\alpha}\left(\int_{\tau_{0}}^{\tau} \frac{s^{2}}{r^{1 / \alpha}(s)} d s\right)^{\alpha+1} Q(\tau)>\frac{\alpha}{4 k_{1}}
$$




$$
\liminf _{\tau \rightarrow \infty}\left(\tau-\tau_{0}\right)^{2} \int_{\tau}^{\infty}\left[\frac{1}{r(v)} \int_{v}^{\infty} \int_{a}^{b} q(s, \xi) \frac{g^{\alpha}(s, \xi)}{s^{\alpha}} d \sigma(\xi) d s\right]^{1 / \alpha} d v>\frac{1}{4 k_{1}}
$$

and

$$
\liminf _{\tau \rightarrow \infty} \frac{Q(\tau)}{R_{2}(\tau)}\left(\int_{\tau_{0}}^{\tau} R_{2}(s) d s\right)^{\alpha+1}>\frac{\alpha}{4 k_{1}}
$$

for some $\mu_{1} \in(0,1)$, then every solution of $(1.1)$ is oscillatory.

Example 2.1 Consider the fourth-order differential equation

$$
\left(e^{3 \tau}\left(x^{\prime \prime \prime}(\tau)\right)^{3}\right)^{\prime}+\int_{0}^{1} \frac{\delta \tau e^{\tau(\xi+3)}}{e^{\tau}-1} x^{3}(\tau-\xi) d \xi
$$

where $\delta>0$ is a constant. We note that

$$
R_{i}(\tau)=e^{-\tau}, \quad i=1,2,3 \text { and } Q(\tau)=\delta e^{3 \tau}
$$

If we choose $\rho(\tau)=\vartheta(\tau)=1$ and $k_{1}=1$, then it easy to see that conditions (2.1), (2.2), (2.3) and (2.4) hold for $\delta>\frac{81}{256}$. Thus, from Theorem 2.1, every solution of equation (2.42) is oscillatory for $\delta>\frac{81}{256}$.

Example 2.2 Consider the delay differential equation

$$
\left(e^{\tau} x^{\prime \prime \prime}(\tau)\right)^{\prime}+b e^{\tau} x(\tau-1)=0, \quad \tau \geq 1
$$

where $b>0$. According to Corollary 4 in [2], equation (2.43) is oscillatory if $b>\frac{2^{5}}{e}$. If we choose $\rho(\tau)=\vartheta(\tau)=1$ and $k_{1}=1$, then we conclude that (2.1) and (2.2) are satisfied and (2.3) and (2.4) hold for $b>\frac{1}{4}$. Hence, by Theorem 2.1, every solution of equation (2.43) is oscillatory for $b>\frac{1}{4}$. Then our results supplement and improve some results obtained in [2]. In particular, we consider the equation

$$
\left(e^{\tau} x^{\prime \prime \prime}(\tau)\right)^{\prime}+25 \sqrt{2} e^{\tau+2 \gamma} x(\tau-\gamma)=0, \quad \tau \geq \gamma
$$

where $\gamma=\sin ^{-1} \frac{7}{5 \sqrt{2}}$. Since $b=25 \sqrt{2} e^{2 \gamma}>\frac{1}{4}$, every solution of equation (2.44) is oscillatory. For example, $x(\tau)=e^{2 \tau} \sin (\tau)$ is a solution of equation (2.44). On the other hand, [24] showed that every nonoscillatory solution of

$$
\left(e^{\tau} x^{\prime \prime \prime}(\tau)\right)^{\prime}+\frac{e^{\tau-1 / 2}}{16} x(\tau-1)=0, \quad \tau \geq 1
$$

tends to zero as $\tau \rightarrow \infty$, and we note that $b=\frac{e^{-1 / 2}}{16}<\frac{1}{4}$.

\section{Acknowledgements}

The authors express their sincere gratitude to the editors and the anonymous referee for careful reading of the original manuscript and useful comments. 
Authors' contributions

All authors read and approved the final manuscript.

\section{Author details}

'Department of Mathematics, Faculty of Science, Mansoura University, Mansoura, 35516, Egypt. ${ }^{2}$ Department of Mathematics, Hadhramout University, Hadhramout, Yemen.

\section{Publisher's Note}

Springer Nature remains neutral with regard to jurisdictional claims in published maps and institutional affiliations.

Received: 11 July 2017 Accepted: 8 August 2017 Published online: 31 August 2017

\section{References}

1. Agarwal, RP, Grace, SR, O'Regan, D: Oscillation Theory for Difference and Functional Differential Equations. Kluwer Academic, Dordrecht (2000)

2. Baculıkova, B, Dzurina, J, Graef, JR: On the oscillation of higher-order delay differential equations. J. Math. Sci. 187(4) $387-400(2012)$

3. Dzurina, J, Stavroulakis, IP: Oscillation criteria for second-order delay differential equations. Appl. Math. Comput. 140, 445-453 (2003)

4. Elabbasy, EM, Moaaz, O, Almehabresh, ES: Oscillation properties of third order neutral delay differential equations. Appl. Math. (Irvine) 7, 1780-1788 (2016)

5. Elabbasy, EM, Moaaz, O, Bazighifan, O: Oscillation criteria for fourth-order nonlinear differential equations. Int. J. Mod. Math. Sci. 15(1), 50-57 (2017)

6. Elabbasy, EM, Barsoum, MY, Moaaz, O: Boundedness and oscillation of third order neutral differential equations with deviating arguments. J. Appl. Math. Phys. 3, 1367-1375 (2015)

7. Elabbasy, EM, Moaaz, O: On the oscillation of third order neutral differential equations. Asian J. Math. Appl. 2016, ama0274 (2016)

8. Elabbasy, EM, Moaaz, O: New oscillation results for class of third order neutral delay differential equations with distributed deviating arguments. Glob. J. Sci. Front. Res. 15(9), Version 1.0 (2015)

9. Elabbasy, EM, Moaaz, O: Oscillation criteria for third order nonlinear neutral differential equations with deviating arguments. Int. J. Comput. Sci. Manag. Res. 5(1) (2016)

10. Elabbasy, EM, Moaaz, O: On the asymptotic behavior of third-order nonlinear functional differential equations Serdica Math. J. 42(2), 157-174 (2016)

11. Erbe, LH, Kong, Q, Zhang, BG: Oscillation Theory for Functional Differential Equations. Dekker, New York (1995)

12. Grace, SR, Bohner, M, Liu, A: Oscillation criteria for fourth-order functional differential equations. Math. Slovaca 63(6), 1303-1320 (2013)

13. Gyori, I, Ladas, G: Oscillation Theory of Delay Differential Equations with Applications. Clarendon, Oxford (1991)

14. Hale, JK: Theory of Functional Differential Equations. Springer, New York (1997)

15. Kiguradze, IT, Chanturia, TA: Asymptotic Properties of Solutions of Nonautonomous Ordinary Differential Equations. Kluwer Academic, Dordrecht (1993)

16. Li, T, Baculíková, B, Džurina, J, Zhang, C: Oscillation of fourth-order neutral differential equations with p-Laplacian like operators. Bound. Value Probl. 2014, 56 (2014)

17. Li, T, Han, Z, Zhao, P, Sun, S: Oscillation of even-order neutral delay differential equations. Adv. Differ. Equ. 2010 $184180(2010)$

18. Li, T, Thandapani, E, Tang, S: Oscillation theorems for fourth-order delay dynamic equations on time scales. Bull. Math. Anal. Appl. 3, 190-199 (2011)

19. Li, T, Zhang, C, Thandapani, E: Asymptotic behavior of fourth-order neutral dynamic equations with noncanonical operators. Taiwan. J. Math. 18(4), 1003-1019 (2014). doi:10.11650/tjm.18.2014.2678

20. Liu, XZ, Fu, XL: Nonlinear differential inequalities with distributed deviating argument and applications. Nonlinear World 4, 409-427 (1994)

21. Moaaz, O: Oscillation Theorems for Certain Second Order Differential Equations. LAP LAMBERT Academic Publishing (2014)

22. Tripathy, AK, Panigrahi, S, Basu, R: Oscillation results for fourth-order nonlinear neutral differential equations with positive and negative coefficients. J. Math. Sci. 194(4), 453-471 (2013)

23. Tripathy, AK: Oscillation criteria for a class of nonlinear fourth order neutral differential equations. Math. Slovaca 63(2), 243-262 (2013)

24. Zhang, C, Li, T, Sun, B, Thandapani, E: On the oscillation of higher-order half-linear delay differential equations. Appl. Math. Lett. 24, 1618-1621 (2011)

25. Zhang, C, Li, T, Saker, SH: Oscillation of fourth-order delay differential equation. J. Math. Sci. 201(3), 296-309 (2014) 